Pacific

Journal of

Mathematics

\title{
DIMENSION ESTIMATE OF HARMONIC FORMS ON COMPLETE MANIFOLDS
}

Jui-Tang Ray Chen and Chiung-Jue Anna Sung 


\title{
DIMENSION ESTIMATE OF HARMONIC FORMS ON COMPLETE MANIFOLDS
}

\author{
Jui-TAng RAy Chen And Chiung-Jue AnNa Sung
}

\begin{abstract}
We consider the space of polynomial-growth harmonic forms. We prove that the dimension of such spaces must be finite and can be estimated if the metric is uniformly equivalent to one with asymptotically nonnegative curvature operator. This implies that the space of harmonic forms of polynomial growth order on the connected sum manifolds with nonnegative curvature operator must be finite-dimensional, which generalizes work of Tam.
\end{abstract}

\section{Introduction}

Let $\left(M^{m}, g\right)$ be an $m$-dimensional manifold with complete Riemannian metric $g$, where $m \geq 3$. We assume that the curvature operator of $M$ is asymptotically nonnegative and we focus on the space of polynomial-growth harmonic $p$-forms of degree at most $d$ on the manifold. Classical de Rham-Hodge theory implies that in the compact case the dimension of the space of harmonic forms is a topological invariant of the manifold, hence independent of the choice of the Riemannian metric. For complete noncompact manifolds, this topological invariance is no longer true. Nonetheless, it is an important question to study the space of harmonic forms and to seek topological and geometrical links. Yau [1975] proved that any positive harmonic function on a manifold with nonnegative Ricci curvature must be constant; hence the strong Liouville property holds. Saloff and Coste [1992] extended the result to the case where any Riemannian metric $g^{\prime}$ is uniformly equivalent to $g$. Thus, the space of positive harmonic functions is stable under a quasi-isometry for $(M, g)$.

A complete manifold $M$ is said to satisfy a Sobolev inequality $S(A, v)$ if there exist a point $q \in M$ and constants $A>0, v>2$, such that for all $r>0$ and all $f \in C_{0}^{\infty}\left(B_{q}(r)\right)$, we have

$$
\int_{B_{q}(r)}|f|^{2 v /(v-2)} \leq A r^{2} V(q, r)^{-2 / v} \int_{B_{q}(r)}\left(|\nabla f|^{2}+r^{-2} f^{2}\right),
$$

MSC2000: primary 58A05; secondary 58A 10.

Keywords: harmonic forms, curvature operator.

Sung was partially supported by the NSC.. 
where $V(q, r)$ is the volume of the geodesic ball $B_{q}(r)$. Examples include minimal submanifolds with Euclidean volume growth in $\mathbb{R}^{m}$ and manifolds with a nonnegative Ricci curvature. If a manifold satisfies a Sobolev inequality when endowed with a certain complete Riemannian metric, it obviously satisfies such an inequality (possibly with a different $A$ ) for any uniformly equivalent metric.

$\mathrm{Li}$ and Wang [1999], extending earlier work of $\mathrm{Li}$ [1997], proved that the dimension of the space $H_{d}^{0}(M)$ of polynomial-growth harmonic functions of growth order at most $d$ has an estimate

$$
\operatorname{dim} H_{d}^{0}(M) \leq C(A, v) d^{v}
$$

provided that the underlying manifold satisfies the Sobolev inequality $S(A, v)$. So the finite dimensionality of the space $H_{d}^{0}(M)$ is valid on such a manifold with respect to any uniformly equivalent metric.

Concerning general harmonic $p$-forms, Li [1997] established a dimension estimate of the space of polynomial-growth harmonic forms. Assuming that $K_{p}$, defined as the curvature operator on $M$ if $p \geq 1$, is nonnegative, Li proved that

$$
\operatorname{dim} H_{d}^{p}(M) \leq C d^{m-1},
$$

where $H_{d}^{p}(M)$ denotes the space of polynomial-growth harmonic $p$-forms on $M$ of growth order at most $d$. Recently, Chen and Sung [2006] showed that the stability of finite dimensionality of the space of $H_{d}^{p}(M)$ holds true under any uniformly equivalent metric on such manifold $M$.

Interestingly, Tam [1998] proved that if $M$ is a complete manifold with nonnegative Ricci curvature outside a compact set, and if each unbounded component of $M \backslash D$, where $D$ is a compact smooth domain in $M$, satisfies a certain kind of volume comparison property, then the space of polynomial-growth harmonic functions of degree at most $d$ is finite dimensional. Furthermore, he proves the finite dimensionality of the space of polynomial-growth harmonic forms with a fixed growth rate on manifolds with asymptotically nonnegative curvature operator and the volume comparison property. The curvature operator $K_{p}$ of $M$ is asymptotically nonnegative if $K_{p} \geq-K(r)$, where $K(r):[0, \infty) \rightarrow[0, \infty)$ is a nonnegative nonincreasing continuous function of distance $r$ to a fixed point $q \in M$ which satisfies the integrability condition

$$
\int_{0}^{\infty} r K(r)<\infty
$$

In view of the preceding results on the space $H_{d}^{p}(M)$, one would naturally to ask if the dimension of the space $H_{d}^{p}(M)$ is stable under a uniformly equivalent metric on $M$ with asymptotically nonnegative curvature operator. An objective of 
this paper is to establish that the dimension of the space $H_{d}^{p}(M)$ remains finite under a uniformly equivalent metric on $M$.

It is proved in [ $\mathrm{Li}$ and Tam 1992] that if the Ricci curvature of $M$ is asymptotically nonnegative then $M$ has finitely many ends. Also, by [Li and Tam 1995, Proposition 3.8], the volume comparison condition holds on $M$ if $M$ is a complete noncompact manifold with asymptotically nonnegative sectional curvature. However, it remains an open question whether an end of $M$ will satisfy the volume comparison condition, if we only assume that $M$ has nonnegative Ricci curvature outside a compact set.

Main Theorem 1.1. Let $\left(M^{m}, g\right)$ be a complete Riemannian manifold. Suppose that the curvature operator $K_{p}$ is asymptotically nonnegative on $(M, g)$ and the metric $g^{\prime}$ is uniformly equivalent to $g$ on $M$. Then there exist constants $C>0$ and $v>2$ such that the dimension of the space $H_{d}^{p}\left(M, g^{\prime}\right)$ is finite and satisfies the inequality

$$
\operatorname{dim} H_{d}^{p}\left(M, g^{\prime}\right) \leq C d^{v}
$$

for all $d \geq 1, p>1$.

Remark 1.2. For $p=1$, the curvature operator becomes Ricci curvature on $M$, we must assume that the first Betti number of $M$ is finite so that the volume comparison condition holds true; compare [Li and Tam 1995]. Under this assumption, the theorem is valid.

An immediate consequence is that the space of polynomial-growth harmonic forms on the connected sum manifolds with nonnegative curvature operator must be finite-dimensional under quasi-isometry. Moreover,

Corollary 1.3. Let $(M, g)$ be a complete Riemannian manifold has nonnegative curvature operator outside a compact set, with finite first Betti number. If the metric $g^{\prime}$ is uniformly equivalent to $g$ on $M$. Then there exist constants $C>0$ and $v>2$ such that the dimension of the space $H_{d}^{p}\left(M, g^{\prime}\right)$ is finite and satisfies the inequality

$$
\operatorname{dim} H_{d}^{p}\left(M, g^{\prime}\right) \leq C d^{v}
$$

for all $d \geq 1, p \geq 1$.

We say $g^{\prime}$ is uniformly equivalent to $g$ if there is some positive constant $c$ such that $c^{-1} g^{\prime} \leq g \leq c g^{\prime}$ in the sense of bilinear forms. In other words, the Riemannian manifolds $(M, g)$ and $\left(M, g^{\prime}\right)$ are then called quasi-isometric. Clearly, quantities such as the distance and volume are uniformly equivalent under quasi-isometry; in particular, a Riemannian manifold quasi-isometric to a complete manifold is also complete. However, in general, any quantity involving derivatives of the Riemannian metric will not be comparable under a quasi-isometry; in particular, the same curvature condition is not expected to hold under a uniformly equivalent change 
of the metric. We overcome the difficulty by relating the space of harmonic forms to the eigenvalues of the Hodge Laplacian on the Busemann balls of the manifold with respect to the absolute boundary conditions. This idea was first introduced and successfully pursued in [Li and Wang 1999] for the harmonic functions. Here, our additional steps are to give the lower bound estimate of the eigenvalue of $p$ form on Busemann balls of $M$, also show that such eigenvalues on each end of manifold are comparable under a uniformly equivalent change of the metric. We obtain a lower bound estimate of eigenvalue by modifying an argument from [Li 1980] in Section 2. Our main result is then proved in Section 3.

Throughout the paper, we assume that the first Betti number of $M$ is finite for the case of $p=1$.

\section{Eigenvalue estimates}

Let $\left(M^{m}, g\right)$ be a complete, oriented Riemannian manifold with dimension $m$. The Hodge-Laplace-Beltrami operator $\Delta$ acting on the space of smooth $p$-forms $\Lambda^{p}(M)$ is defined as

$$
\Delta=d \delta+\delta d,
$$

here $d$ denotes the exterior differential operator and $\delta=* d *$, where the linear operator $*$ is defined point-wise by

$$
*\left(w_{1} \wedge \cdots \wedge w_{p}\right)=w_{p+1} \wedge \cdots \wedge w_{m}
$$

for a positively oriented orthonormal coframe $\left\{w_{1}, w_{2}, \ldots, w_{m}\right\}$ at the point. A $p$-form $w \in \Lambda^{p}(M)$ is called a harmonic $p$-form on $(M, g)$ if

$$
\Delta_{g} w=0 .
$$

Let $q$ denote a point on $(M, g)$ and let $r_{q}(x)$ represent the geodesic distance function from $x \in M$ to the point $q$. For each $d \geq 0$, we denote the space of polynomialgrowth harmonic $p$-forms of degree at most $d$ by

$$
H_{d}^{p}(M, g) \equiv\left\{w \in \Lambda^{p}(M) \mid \Delta_{g} w=0, \text { and }|w|=O\left(r_{q}^{d}\right)\right\} .
$$

For a bounded smooth domain $B \subset M$, a $p$-form $w$ is said to satisfy the absolute condition on $B$ if the tangential component of both $w$ and $\delta w$ on the boundary $\partial B$ are zeros. On the boundary $\partial B$, let $N_{\partial B}$ (respectively $N_{\partial B_{q}}^{*}$ ) represent the inward unit normal vector (respectively covector) field. Now, denote exterior multiplication by $\operatorname{ext}(\cdot)$ and dual exterior multiplication by $\operatorname{int}(\cdot)$. It is not difficult to verify that $\Delta$ is a self-adjoint nonnegative operator on the space $\Lambda^{p}(B)$ of smooth $p$-forms on $B$ satisfying the absolute boundary condition. By the standard elliptic theory, we see that $\Delta$ has a countable set of eigenvalues and the multiplicity of each eigenvalue is finite. If we list all the eigenvalues with multiplicity in nondecreasing order by 
$\left\{\lambda_{k}, k=1,2,3, \ldots\right\}$, then $\lambda_{k} \rightarrow \infty$ as $k \rightarrow \infty$. Moreover, the $i$-th eigenvalue can be characterized as

$$
\lambda_{i}=\inf _{\operatorname{dim} V=i} \sup _{w \in V \backslash\{0\}} R(w),
$$

where $V$ is a subspace of $\Lambda^{p}(B)$ and the Rayleigh-Ritz quotient $R(w)$ is defined by

$$
R(w)=\frac{(d w, d w)+(\delta w, \delta w)}{(w, w)}
$$

for $w \in \Lambda^{p}(B)$ and the $L^{2}$ inner product for two forms $v$ and $w$ in $\Lambda^{p}(B)$ is defined by

$$
(v, w)=\int_{B}\langle v, w\rangle d x
$$

with $\langle v, w\rangle$ being the point-wise inner product between $v$ and $w$.

On the other hand, the Hodge-de Rham theorem provides an orthogonal decomposition of the space $\Lambda^{p}(B)$ of differential forms of degree $p$ on $B$. For any $w \in \Lambda^{p}(B), w$ can be uniquely written as

$$
w=h+d v+\delta u,
$$

where $h \in H_{p}(B)$, the space of harmonic $p$-forms satisfying the absolute boundary condition and $v \in \Lambda^{p-1}(B), u \in \Lambda^{p+1}(B)$. Clearly, the operator $\Delta$ leaves this decomposition invariant, and the eigenvalues of $\Delta$ on the subspace $H_{p}(B)$ are zeros.

Denote by $\left\{\mu_{j}^{e}(g) \mid j \geq 1\right\}$ the eigenvalues of $\Delta$ acting on the subspace $d \Lambda^{p-1}(B)$ of exact $p$-forms, and by $\left\{\mu_{l}^{c o}(g) \mid l \geq 1\right\}$ those corresponding to the subspace $\delta \Lambda^{p+1}(B)$ of coexact $p$-forms. Then the eigenvalues $\left\{\lambda_{i}(g) \mid i>\operatorname{dim} H_{p}(B)\right\}$ is equal to the reordered union of $\left\{\mu_{j}^{e}(g) \mid j \geq 1\right\}$ and $\left\{\mu_{l}^{c o}(g) \mid l \geq 1\right\}$. We have,

$$
\left\{\lambda_{i}(g) \mid i>\operatorname{dim} H_{p}(B)\right\}=\left\{\mu_{j}^{e}(g) \mid j \geq 1\right\} \cup\left\{\mu_{l}^{c o}(g) \mid l \geq 1\right\} .
$$

The next lemma is essentially due to [Dodziuk 1982]; a proof is given in [Chen and Sung 2006].

Lemma 2.1. Let $(M, g)$ be a complete manifold with Riemannian metric $g$. Let $g^{\prime}$ denote another Riemannian metric on $M$ which is uniformly equivalent to $g$. Then

$$
\operatorname{dim} H_{p}(B, g)=\operatorname{dim} H_{p}\left(B, g^{\prime}\right),
$$

and there exists a positive constant $C$ such that

$$
C^{-1} \lambda_{i}(g) \leq \lambda_{i}\left(g^{\prime}\right) \leq C \lambda_{i}(g)
$$

for all $i \geq 1$. 
We will obtain a lower bound estimate of the eigenvalues of $p$-forms satisfying the absolute boundary condition on Busemann balls of a manifold with asymptotically nonnegative curvature operator. The argument closely follows those in [ $\mathrm{Li}$ 1980] and [Chen and Sung 2006].

An end $E$ of a manifold $M$ is an unbounded component of the complement of some compact smooth subset $D$ of $M$. In this case, $E$ is called an end corresponding to $D$. We say that $M$ has finitely many ends if there exists $b<\infty$ such that the number of ends corresponding to $D$ is less than or equal to $b$ for any compact subset $D \subset M$. Let $E_{1}, E_{2}, \ldots, E_{L}$ be the ends of $M$ with respect to $D$. We say that $E$ satisfies volume comparison property if there exists a constant $\zeta>0$ such that

$$
V_{E}(r) \leq \zeta V_{x}\left(\frac{r}{2}\right)
$$

for all $x \in \partial B_{E}(r)$ and for $r$ large enough. Here we use $B_{E}(r)$ to denote $B_{q}(r) \cap E$, $\partial B_{E}(r)=\partial B_{q}(r) \cap E$, and $V_{E}(r)$ is the volume of $B_{E}(r)$. Also, denote

$$
B_{E}\left(r_{1}, r\right)=B_{E}(r) \backslash B_{E}\left(r_{1}\right),
$$

and

$$
\partial B_{E}\left(r_{1}, r\right)=\partial B_{E}(r) \cup \partial B_{E}\left(r_{1}\right),
$$

where $r_{1} \leq r$. By [Tam 1998, Lemma 1.1], the volume doubling property holds on ends $\left\{E_{l}\right\}_{l=1}^{L}$ of $M$, that is for $r>2 r_{0}$,

$$
V_{E_{l}}((1+\varepsilon) r) \leq(1+\varepsilon)^{\mu} \quad V_{E_{l}}(r)
$$

where $\mu>0$ is a constant depending only on $m$. Moreover, given any $\eta>0$, there is $r_{1}>2 r_{0}$ such that for all $x \in \partial B_{E}(R)$, with $R>r_{1}$, and for all $\frac{3}{4} R>r^{\prime}>r>0$, we have

$$
V_{x}\left(r^{\prime}\right) \leq\left(\frac{r^{\prime}}{r}\right)^{n+\eta} V_{x}(r) .
$$

Let $\gamma:[0, \infty) \rightarrow M$ be a ray with $\gamma(0)=q$, a fixed point in $M$; namely, $\gamma$ is a geodesic of $(M, g)$ and the geodesic distance $r(\gamma(t), \gamma(s))$ between $\gamma(t)$ and $\gamma(s)$ is equal to $|t-s|$ for all $t$ and $s$ in $[0, \infty)$. We define $b_{t}(x)=t-r(x, \gamma(t))$ for $t \geq 0$. For any fixed $x, b_{t}(x)$ is a nondecreasing function of $t$ and $b_{t}(x)=$ $r(q, \gamma(t))-r(x, \gamma(t)) \leq r(q, x)$. Therefore $b_{\gamma}(x)=\lim _{t \rightarrow \infty} b_{t}(x)$ exists for all $x \in M$. In fact, $b_{t}(x)$ converges uniformly on compact sets to $b_{\gamma}(x)$. Set

$$
\beta(x)=\sup \left\{b_{\gamma}(x) \mid \gamma \text { is a ray from } p\right\} .
$$

Since for each $x$ and for any ray $\gamma, b_{\gamma}(x) \leq r(x, p), \beta(x)$ is well defined and finite. We call $\beta(x)$ the Busemann function of $(M, g)$ (based at point $p$ ). 
We define $B(a)=\{x \mid \beta(x) \leq a\}$ as a Busemann ball on $M$, for some positive constant $a$. It is well known that $\beta(x)$ is proper and convex if the curvature operator of $M$ is nonnegative. Therefore, the boundary of the Busemann ball is convex. Denote a Busemann ball on end $E$ as $B_{E}(a)=B(a) \cap E$. The boundary of the Busemann ball $B_{E}(a)$ is convex if the curvature operator is nonnegative on each end. We will obtain a lower bound estimate of the eigenvalues of $p$-forms satisfying the absolute boundary condition on Busemann balls of a manifold with asymptotically nonnegative curvature operator. The argument closely follows those in [Li 1980] and [Chen and Sung 2006].

Lemma 2.2. Let $M^{m}$ be a complete manifold with asymptotically nonnegative curvature operator. If $E$ is an end of $M$ with respect to a compact subset $B_{q}\left(r_{0}\right) \subset$ $M, r_{0}>0$, for a large enough $r>2 r_{0}$, such that $B_{q}\left(r_{0}\right)$ is contained in Busemann ball $B(r)$, then there exist constants $C>0$ and $v>2$ such that

$$
\operatorname{dim} H_{p}(B(r)) \leq C
$$

and, for each $k>\operatorname{dim} H_{p}(B(r))$, there exists a constant $C$ depending only on $v$, $m, p$ and $\eta$ such that

$$
\lambda_{k}(B(r)) \geq C k^{2 / v} r^{-2} .
$$

Proof. Let $\mathscr{V}$ be the $k$-dimensional space spanned by the eigen $p$-forms corresponding to the first $k$ eigenvalues $\left\{\lambda_{1}, \ldots, \lambda_{k}\right\}$ on $B(r)$. Then there exists $w \in \mathscr{V}$, $w \neq 0$, such that

$$
\frac{k}{V}\|w\|_{2}^{2} \leq\|w\|_{\infty}^{2} \cdot \min \left\{\left(\begin{array}{c}
m \\
p
\end{array}\right), k\right\},
$$

where $V=V(B(r))$ denotes the volume of $B(r)$. This is a result in [Li 1980].

On the other hand, we claim that there exist constants $C>0, k_{0}>0$ and $v>2$ such that for $w \in \mathscr{V}$,

$$
\|w\|_{\infty}^{2} \leq C V^{-1} r^{-v} \lambda_{k}^{v / 2}\|w\|_{2}^{2}
$$

for all $k \geq k_{0}$. It is easy to see that the Lemma follows by combining inequalities (4) and (5). To prove (5), by the convexity of Busemann function and [Donnelly and Li 1982, Lemma 6.2], we first observe that for $w \in \Lambda^{p}(B(r))$,

$$
\frac{\partial|w|^{2}}{\partial n} \leq 0
$$

on $\partial B(r)$, where $\partial / \partial n$ is the outward unit normal of $\partial B(r)$. Since curvature operator $K_{p}$ is asymptotically nonnegative, it means $K_{p} \geq-K(r)$, where $K(r)$ : $[0, \infty) \rightarrow[0, \infty)$ is a nonnegative nonincreasing continuous function of distance 
$r$ to a fixed point $q \in M$ which satisfies the integrability condition

$$
\int_{0}^{\infty} r K(r)<\infty
$$

By the argument in [Saloff-Coste 1992], we obtain a local weak Poincaré inequality on the Busemann ball $B(r)$. Also, using doubling volume condition and the local weak Poincaré inequality for the Busemann ball $B(r)$, we have the SobolevPoincaré inequality on $B(r)$ (see [Hajłasz and Koskela 1995])

$$
\left(\int_{B(r)}\left|f-f_{B}\right|^{2 v /(v-2)}\right)^{(v-2) / v} \leq A V^{-2 / v} r^{2} \int_{B(r)}|\nabla f|^{2},
$$

where $v>2, f_{B}=V^{-1}(B(r)) \int_{B(r)} f, A>1$ is a constant depending only on $m$ and $\eta$, and $V=V(B(r))$ is the volume of $B(r)$. Moreover, by [Li 1980], we observe that the Neumann Sobolev-type inequality

$$
\left(\int_{B(r)}|f|^{2 v /(v-2)}\right)^{(v-2) / v} \leq A V^{-2 / v} r^{2}\left(\int_{B(r)}|\nabla f|^{2}+r^{-2} \int_{B(r)}|f|^{2}\right)
$$

holds on $B(r)$.

Let $\left\{w_{i}\right\}_{i=1}^{k}$ be the $p$-eigenforms satisfying the absolute boundary condition with the corresponding nonzero eigenvalues $\left\{\lambda_{i}\right\}_{i=1}^{k}$ and we also assume $\left\{w_{i}\right\}_{i=1}^{k}$ are orthonormal and $\operatorname{span} \mathscr{V}$. If $w \in \mathscr{V}$, then there exist $\left\{a_{i}\right\}_{i=1}^{k}$ such that $w=\sum_{i=1}^{k} a_{i} w_{i}$, that is, $\Delta w=\sum_{i=1}^{k} \lambda_{i} a_{i} w_{i}$. By the Bochner formula,

$$
\frac{1}{2} \Delta|w|^{2} \leq\langle\Delta w, w\rangle-|\nabla w|^{2}+K|w|^{2},
$$

where $K$ is the lower bound of curvature for all $x$ in $B(r)$. Using the fact in [Li 1980, Lemma 8],

$$
|\nabla| w||^{2} \leq|\nabla w|^{2}
$$

Thus,

$$
\frac{1}{2} \Delta|w|^{2} \leq\langle\Delta w, w\rangle-|\nabla| w||^{2}+K|w|^{2}
$$

Let $\alpha \geq 1$, and we multiply both sides of this inequality ((7)) by $|w|^{2 \alpha-2}$ and integrate over $B(r)$,

$$
\begin{aligned}
& \frac{1}{2} \int_{B(r)}|w|^{2 \alpha-2} \Delta|w|^{2} \\
& \quad \leq \int_{B(r)}|w|^{2 \alpha-2}\langle\Delta w, w\rangle+\int_{B(r)} K|w|^{2 \alpha}-\int_{B(r)}|w|^{2 \alpha-2}|\nabla| w||^{2} .
\end{aligned}
$$


Using the absolute boundary condition, the left-hand side of this inequality becomes

$$
\begin{aligned}
\frac{1}{2} \int_{B(r)}|w|^{2 \alpha-2} \Delta|w|^{2} & \geq(\alpha-1) \int_{B(r)}|w|^{2 \alpha-3}\left\langle\nabla|w|, \nabla|w|^{2}\right\rangle \\
& =\frac{2(\alpha-1)}{\alpha^{2}} \int_{B(r)}\left\langle\nabla|w|^{\alpha}, \nabla|w|^{\alpha}\right\rangle,
\end{aligned}
$$

and the third term in the right-hand side of (8) can be rewritten as

$$
\left.\int_{B(r)}|w|^{2 \alpha-2}|\nabla| w\right|^{2}=\left.\left.\frac{1}{\alpha^{2}} \int_{B(r)}|\nabla| w\right|^{\alpha}\right|^{2} .
$$

Hence, (8) becomes

$$
\text { (9) } \begin{aligned}
\frac{2(\alpha-1)}{\alpha^{2}} \int_{B(r)} & \left.\left.|\nabla| w\right|^{\alpha}\right|^{2} \\
\leq & \int_{B(r)}|w|^{2 \alpha-2}\langle\Delta w, w\rangle+\int_{B(r)} K|w|^{2 \alpha}-\left.\left.\frac{1}{\alpha^{2}} \int_{B(r)}|\nabla| w\right|^{\alpha}\right|^{2} .
\end{aligned}
$$

With $f=|w|$, this can be rewritten as

$$
\frac{2 \alpha-1}{\alpha^{2}} \int_{B(r)}\left|\nabla f^{\alpha}\right|^{2} \leq \int_{B(r)} f^{2 \alpha-2}\langle\Delta w, w\rangle+\int_{B(r)} K f^{2 \alpha} .
$$

Applying Neumann Sobolev-type inequality (6) to the function $f^{\alpha}$, one has

$$
\left(\int_{B(r)}|f|^{2 \beta \alpha}\right)^{1 / \beta} \leq A V^{-2 / v} r^{2}\left(\int_{B(r)}\left|\nabla f^{\alpha}\right|^{2}+r^{-2} \int_{B(r)} f^{2 \alpha}\right),
$$

where $\beta=\frac{v}{v-2}$. Thus (10) and (11) suggest

$$
\begin{aligned}
\|f\|_{2 \alpha \beta}^{2 \alpha} \leq \frac{\alpha^{2}}{2 \alpha-1} A V^{-2 / v} & r^{2} \int_{B(r)} f^{2 \alpha-2}\langle\Delta w, w\rangle \\
& +A V^{-2 / v} r^{2}\left(\frac{\alpha^{2}}{2 \alpha-1} \int_{B(r)} K f^{2 \alpha}+r^{-2}\|f\|_{2 \alpha}^{2 \alpha}\right) .
\end{aligned}
$$

By the Hölder inequality, we have

$$
\begin{aligned}
\int K f^{2 \alpha} & \leq B V^{1 / q}\left(\int\left(f^{2 \alpha}\right)^{q /(q-1)}\right)^{(q-1) / q} \\
& \leq B V^{1 / q}\left(\int f^{2 \alpha}\right)^{\frac{\beta(q-1)-q}{q(\beta-1)}}\left(\int f^{2 \alpha \beta}\right)^{\frac{1}{q(\beta-1)}}
\end{aligned}
$$


where $q>\frac{\beta}{\beta-1}=\frac{v}{2}$ and $B=\left(V^{-1} \int_{B(r)}|K|^{q}\right)^{1 / q}$. However, applying the inequality

$$
h^{\varepsilon} \leq \delta_{1}^{(\varepsilon-1) / \varepsilon} h+\delta_{1} \varepsilon^{1 /(1-\varepsilon)}\left(\frac{1}{\varepsilon}-1\right)
$$

by setting $\varepsilon=\frac{\beta(q-1)-q}{q(\beta-1)}$ and

$$
h=\left(\frac{\alpha^{2}}{2 \alpha-1} A B V^{(\nu-2 q) / q v} r^{2}\right)^{\frac{q(\beta-1)}{\beta(q-1)-q}}\left(\int f^{2 \alpha}\right)\left(\int f^{2 \alpha \beta}\right)^{\frac{-1}{\beta}} \text {. }
$$

Then

$$
\begin{array}{rl}
\frac{\alpha^{2}}{2 \alpha-1} A & B V^{(\nu-2 q) / q v} r^{2}\left(\int f^{2 \alpha}\right)^{\frac{\beta(q-1)-q}{q(\beta-1)}}\left(\int f^{2 \alpha \beta}\right)^{\frac{q-\beta(q-1)}{q \beta(\beta-1)}} \\
\leq \delta_{1}^{(\varepsilon-1) / \varepsilon}\left(\frac{\alpha^{2}}{2 \alpha-1} A B V^{(\nu-2 q) / q v} r^{2}\right)^{\frac{2 q}{2 q-v}}\left(\int f^{2 \alpha}\right)\left(\int f^{2 \alpha \beta}\right)^{\frac{-1}{\beta}} \\
+\delta_{1} \varepsilon^{1 /(1-\varepsilon)}\left(\frac{1}{\varepsilon}-1\right) .
\end{array}
$$

If we select $\delta_{1}$ small enough, and since $\frac{\alpha^{2}}{2 \alpha-1} \geq 1$, Equation (12) can be rewritten as

$$
\begin{aligned}
& \|f\|_{2 \alpha \beta}^{2 \alpha} \leq \frac{1}{1-\delta_{1} c(\varepsilon)}\left(\frac{\alpha^{2}}{2 \alpha-1}\right)^{\frac{2 q}{2 q-v}} A V^{-2 / v} r^{2} \\
& \quad \times\left(\int_{B(r)} f^{2 \alpha-2}\langle\Delta w, w\rangle+\left(\delta_{1}^{\nu /(v-2 q)} B^{2 q /(2 q-v)}\left(A r^{2}\right)^{v /(2 q-v)}+\frac{1}{r^{2}}\right)\|f\|_{2 \alpha}^{2 \alpha}\right) .
\end{aligned}
$$

Let $\alpha=\beta^{i}, i=0,1,2, \ldots$ Then

$$
\|f\|_{2 \beta^{i+1}}^{2 \beta^{i}} \leq \tilde{C}\left(\int_{B(r)} f^{2 \beta^{i}-2}\langle\Delta w, w\rangle+\left(\bar{K}+r^{-2}\right)\|f\|_{2 \beta^{i}}^{2 \beta^{i}}\right) .
$$

where

$$
\tilde{C}=\frac{1}{1-\delta_{1} c(\varepsilon)}\left(\frac{\alpha^{2}}{2 \alpha-1}\right)^{\frac{2 q}{2 q-v}} A V^{-2 / v} r^{2} \text { and } \bar{K}=B^{2 q /(2 q-v)}\left(\delta_{1}^{-1} A r^{2}\right)^{v /(2 q-v)} \text {. }
$$

When $i=0,(13)$ gives

$$
\|f\|_{2 \beta}^{2} \leq \frac{1}{1-\delta_{1} c(\varepsilon)} A V^{-2 / v} r^{2}\left(\int_{B(r)}\langle\Delta w, w\rangle+\left(\bar{K}+r^{-2}\right)\|f\|_{2}^{2}\right) .
$$

\section{Since}

$$
\int_{B(r)}\langle\Delta w, w\rangle=\int_{B(r)}\left\langle\lambda_{i} a_{i} w_{i}, a_{j} w_{j}\right\rangle=\lambda_{i} a_{i}^{2} \leq \lambda_{k} a_{i}^{2}=\lambda_{k} \int_{B(r)}\langle w, w\rangle,
$$


this implies

$$
\|f\|_{2 \beta}^{2} \leq \frac{1}{1-\delta_{1} c(\varepsilon)} A V^{-2 / v} r^{2}\left(\lambda_{k}+\bar{K}+r^{-2}\right)\|f\|_{2}^{2} .
$$

By the Hölder inequality,

$$
\|f\|_{2}^{2} \leq V^{(\beta-1) / \beta}\|f\|_{2 \beta}^{2} .
$$

We conclude that

$$
V^{-(\beta-1) / \beta}\|f\|_{2}^{2} \leq \frac{1}{1-\delta_{1} c(\varepsilon)} A V^{-2 / v} r^{2}\left(\lambda_{k}+\bar{K}+r^{-2}\right)\|f\|_{2}^{2} .
$$

Now we claim that for $1 \leq i<\infty$,

$$
\begin{aligned}
& V^{-(\beta-1) / \alpha \beta}\|f\|_{2 \alpha}^{2} \\
& \quad \leq \prod_{j=0}^{i}\left(\frac{\beta^{2 j}}{2 \beta^{j}-1}\right)^{\frac{2 q}{2 q-\nu} \beta^{-j}}\left(\frac{1}{1-\delta_{1} c(\varepsilon)} A V^{-2 / v} r^{2} \lambda_{k}^{*}\right)^{\sum_{j=0}^{i} \beta^{-j}}\|f\|_{2}^{2},
\end{aligned}
$$

where $\lambda_{k}^{*}=\lambda_{k}+\bar{K}+r^{-2}$. Assuming this inequality (14) is true for $\alpha=\beta^{j}$, $j=0, \ldots, i-1$, by induction, we need to show that (14) is still valid for $j=i$. Suppose $g=|\bar{w}|$, where $\bar{w} \in \mathscr{V}$ with the property that

$$
\frac{\|g\|_{2 \alpha}}{\|g\|_{2}} \geq \frac{\|w\|_{2 \alpha}}{\|w\|_{2}} \quad \text { for all } w \in \mathscr{V} .
$$

Without loss of the generality, we may use the scaling and assume $\|g\|_{2}=1$. By the Hölder inequality, Equation (13) implies

$$
\begin{aligned}
\|g\|_{2 \alpha \beta}^{2 \alpha} & \leq \tilde{C}\left(\int_{B(r)} g^{2 \alpha-2}\langle\Delta \bar{w}, \bar{w}\rangle+\left(\bar{K}+r^{-2}\right)\|g\|_{2 \alpha}^{2 \alpha}\right) \\
& \leq \tilde{C}\left(\|g\|_{2 \alpha}^{2 \alpha-1}\|\Delta \bar{w}\|_{2 \alpha}+\left(\bar{K}+r^{-2}\right)\|g\|_{2 \alpha}^{2 \alpha}\right) .
\end{aligned}
$$

We also note that if $s \geq 2$, then there exists a subset $\{\sigma\} \subset\{1,2, \ldots, k\}$ such that

$$
\left\|\sum_{i=1}^{k} \lambda_{i} w_{i}\right\|_{s} \leq\left\|\sum_{\sigma} \lambda_{k} w_{\sigma}\right\|_{s} .
$$

This is proved in [Li 1980, Lemma 17]. Hence, let $\bar{w}=\sum b_{i} w_{i}$, then $\Delta \bar{w}=$ $\sum \lambda_{i} b_{i} w_{i}$ and we have

$$
\begin{aligned}
\|\Delta \bar{w}\|_{2 \alpha} & =\left\|\sum_{i} \lambda_{i} b_{i} w_{i}\right\|_{2 \alpha} \leq\left\|\sum_{\sigma} \lambda_{k} b_{\sigma} w_{\sigma}\right\|_{2 \alpha}=\lambda_{k}\left\|\sum_{\sigma} b_{\sigma} w_{\sigma}\right\|_{2 \alpha} \\
& \leq \lambda_{k}\|g\|_{2 \alpha}\left\|\sum_{\sigma} b_{\sigma} w_{\sigma}\right\|_{2} \text { by (15) } \\
& \leq \lambda_{k}\|g\|_{2 \alpha} .
\end{aligned}
$$


It is obvious that (16) gives

$$
\|g\|_{2 \alpha \beta}^{2 \alpha} \leq \tilde{C} \lambda_{k}^{*}\|g\|_{2 \alpha}^{2 \alpha},
$$

where $\lambda_{k}^{*}=\lambda_{k}+\bar{K}+r^{-2}$. By the method of iteration, we obtain

$$
\|g\|_{2 \alpha \beta}^{2} \leq \tilde{C}_{i}\left(\lambda_{k}^{*}\right)^{\sum_{j=0}^{i} \beta^{-j}}\|g\|_{2}^{2},
$$

where $\alpha=\beta^{i}$ and

$$
\tilde{C}_{i}=\prod_{j=0}^{i}\left(\frac{\beta^{2 j}}{2 \beta^{j}-1}\right)^{2 q /(2 q-v) \beta^{-j}}\left(\frac{1}{1-\delta_{1} c(\varepsilon)} A V^{-2 / v} r^{2}\right)^{\sum_{j=0}^{i} \beta^{-j}} .
$$

On the other hand, by Hölder inequality, we have

$$
\|g\|_{2 \alpha}^{2} \leq V^{(\beta-1) / \alpha \beta}\|g\|_{2 \alpha \beta}^{2} .
$$

Therefore,

$$
V^{-(\beta-1) / \alpha \beta}\|g\|_{2 \alpha}^{2} \leq \tilde{C}_{i} \cdot\left(\lambda_{k}^{*}\right)^{\sum_{j=0}^{i} \beta^{-j}}\|g\|_{2}^{2} .
$$

Applying (15) to (17), it is easy to check that

$$
V^{-(\beta-1) / \alpha \beta}\|f\|_{2 \beta^{i}}^{2} \leq \tilde{C}_{i} \cdot\left(\lambda_{k}^{*}\right)^{\sum_{j=0}^{i} \beta^{-j}}\|f\|_{2}^{2} .
$$

Letting $i \rightarrow \infty$, due to $\sum_{j=0}^{\infty} \beta^{-j}=v / 2$ and

$$
\prod_{j=0}^{\infty}\left(\frac{\beta^{2 j}}{2 \beta^{j}-1}\right)^{\beta^{-j}} \leq \exp \frac{1}{\beta^{1 / 2}-1}=c_{1}(\nu),
$$

we conclude that

$$
\|f\|_{\infty}^{2} \leq C(v, q)\left(A V^{-2 / v} r^{2} \lambda_{k}^{*}\right)^{v / 2}\|f\|_{2}^{2},
$$

where $A$ is a positive constant depending only on $m$ and $\eta$. This means, for all $w \in \mathscr{V}, w$ satisfies

$$
\|w\|_{\infty}^{2} \leq C(\nu, \eta, m) V^{-1} r^{v}\left(\lambda_{k}^{*}\right)^{v / 2}\|w\|_{2}^{2},
$$

for all $q>v / 2$, where

$$
\lambda_{k}^{*}=\lambda_{k}+\bar{K}+r^{-2} \quad \text { and } \quad \bar{K}=\delta_{1}^{\nu /(\nu-2 q)} B^{2 q /(2 q-v)}\left(A r^{2}\right)^{\nu /(2 q-v)} .
$$

In fact, by the assumption of curvature, we have $B \leq 1$; hence

$$
\|w\|_{\infty}^{2} \leq C(v, \eta, m) V^{-1} r^{v}\left(\lambda_{k}+c\left(\delta_{1}, v\right) r^{2 v /(2 q-v)}+r^{-2}\right)^{v / 2}\|w\|_{2}^{2} .
$$

We note that the Hodge Laplace Beltrami operator $\Delta=d \delta+\delta d$ is nonnegative and self-adjoint on $B(r)$ under the absolute boundary condition. Hence, using the 
standard elliptic theory, if we assume $\lambda_{1}$ be the first nonzero eigenvalue, then we have

$$
0<\lambda_{1} \leq \lambda_{2} \leq \cdots \rightarrow \infty
$$

This means, there exists $k_{0}$ large enough such that the $k$-th nonzero eigenvalue

$$
\lambda_{k} \geq \max \left\{c\left(\delta_{1}, v\right) r^{2 v /(2 q-v)}, r^{-2}\right\} \text {. }
$$

Thus, by (18) and (19), we have

$$
\|w\|_{\infty}^{2} \leq C(v, \eta, m) V^{-1} r^{v} \lambda_{k}^{v / 2}\|w\|_{2}^{2}
$$

for all $w$ belong to the space $\mathscr{V}$ spanned by the $p$-eigenforms corresponding to the first $k$ nonzero eigenvalues. Therefore, the dimension estimate (4) gives

$$
\frac{k}{V}\|w\|_{2}^{2} \leq\left(\begin{array}{c}
m \\
p
\end{array}\right)\|w\|_{\infty}^{2} \leq C(v, \eta, m, p) V^{-1} r^{v} \lambda_{k}^{v / 2}\|w\|_{2}^{2},
$$

and we conclude that

$$
\lambda_{k}(B(r)) \geq C k^{2 / v} r^{-2}
$$

for all $k \geq k_{0}$, where $C=C(v, \eta, m, p)$ is a positive constant.

\section{Main Result}

Let $\left(M^{m}, g\right), m \geq 3$, be a complete noncompact manifold with Riemannian metric $g$. We consider the manifold $M$ with its curvature operator $K_{p}(x)$ is asymptotically nonnegative. By [Li and Tam 1992], we know that $M$ has finitely many ends if the curvature operator $K_{p}(x)$ of $M$ is asymptotically nonnegative. Assume $E_{1}, \ldots, E_{L}$ be the ends of $M$ with respect to a compact smooth domain $B_{q}\left(r_{0}\right)$ in $M$. Let $\mathfrak{B}$ be a $n$-dimensional vector bundle over $M$ with a metric. For $r>4 r_{0}>0$, with $B_{q}\left(r_{0}\right) \subset B(r)$, where $B(r)$ is a Busemann ball in $M$. We define a positive semidefinite symmetric bilinear form $S_{r}$ on the space of section $\Gamma(\mathfrak{B})$ of $\mathfrak{B}$ by

$$
S_{r}(u, v)=V^{-1}(r) \int_{B(r)}\langle u, v\rangle
$$

for $u, v \in \Gamma(\mathfrak{B})$. In particular, $S_{r}$ is always positive definite, and $\left(\mathfrak{B}, S_{r}\right)$ is an inner product space in $B(r)$.

Suppose each end satisfies volume comparison property, the volume doubling property holds on ends $\left\{E_{l}\right\}_{l=1}^{L}$ of $M$, that is for $r>2 r_{0}$ and $\varepsilon>0$,

$$
V_{E_{l}}((1+\varepsilon) r) \leq(1+\varepsilon)^{\mu} V_{E_{l}}(r),
$$

where $\mu>0$ is a constant depending only on $m$. Moreover, given any $\eta>0$, there is $r_{1}>2 r_{0}$ such that for all $x \in \partial B_{E}(R)$, with $R>r_{1}$, and for all $\frac{3}{4} R>r^{\prime}>r>0$, 
we have

$$
V_{x}\left(r^{\prime}\right) \leq\left(\frac{r^{\prime}}{r}\right)^{n+\eta} V_{x}(r) .
$$

The curvature operator $K_{p}$ on $M$ is defined by

$$
K_{p} \geq-K= \begin{cases}\text { lower bound of curvature operator } & \text { if } p>1 \\ \text { lower bound of Ricci curvature } & \text { if } p=1\end{cases}
$$

Concerning the asymptotically nonnegative curvature operator, the volume comparison property holds on each end for $p>1$ [Li and Tam 1995, Proposition 3.8]. For the case of $p=1$, the curvature operator is the Ricci curvature, the volume comparison property holds on ends of $M$ if we assume that the first Betti number of $M$ is finite.

Lemma 3.1. Let $\mathscr{V}$ be a $k$-dimensional subspace of a vector space $W$. Assume that $W$ is endowed with an inner production $L$ and a bilinear form $\Phi$. Then for any given linearly independent set of vectors $\left\{w_{1}, \ldots, w_{k-1}\right\} \subset W$, there exists an orthonormal basis $\left\{v_{1}, \ldots, v_{k}\right\}$ of $\mathscr{V}$ with respect to $L$ such that $\Phi\left(v_{i}, w_{j}\right)=0$ for all $1 \leq j<i \leq k$.

Lemma 3.2. Let $M$ be a complete Riemannian manifold with asymptotically nonnegative curvature operator. Let $\mathcal{V}$ be a $k$-dimensional subspace of $H_{d}^{p}(M, g)$, and let $E_{1}, E_{2}, \ldots, E_{L}$ be the ends of $M$ with respect to $B_{q}\left(r_{0}\right), r_{0}>0$. For any fixed $0<\varepsilon<\frac{1}{4}, r>4 r_{0}$ and any subspace $Y$ of $\mathscr{V}$, if $\left\{v_{s+1}, \ldots, v_{k}\right\}$ is an orthonormal basis of inner production $S_{(1+\varepsilon) r}$ on $Y$. Then

$$
\sum_{i=s+1}^{k} S_{r}\left(v_{i}, v_{i}\right) \leq \frac{8(1+\varepsilon)^{\mu}}{\varepsilon^{2} r^{2}} \sum_{i=s+1}^{k} \lambda_{i}^{-1}(B((1+\varepsilon) r)),
$$

where $\mu>0$ is a constant depending only on $m$.

Proof. Let $\lambda_{i}(B((1+\varepsilon) r))$ denote the $i$-th nonzero eigenvalue of $p$-forms on Busemann ball $B((1+\varepsilon) r)$ satisfying the absolute boundary condition on $\partial B((1+\varepsilon) r)$. Let $\phi$ be a nonnegative function defined on $B((1+\varepsilon) r)$ satisfying these conditions:

$$
\begin{aligned}
\phi=1 & \text { on } B(r), \\
0 \leq \phi \leq 1 & \text { on } B((1+\varepsilon) r), \\
\phi=0 & \text { on } \partial B((1+\varepsilon) r),
\end{aligned}
$$

and

$$
|\nabla \phi| \leq \frac{2}{\varepsilon r} .
$$

Observing that by the property of unique continuation, $\mathscr{V}$ is a $k$-dimensional subspace because

$$
\mathscr{V} \subset L^{2}(B((1+\varepsilon) r), \phi d v) \cap L^{2}(B(r), d v) .
$$


Applying Lemma 3.1 with $\left\{w_{1}, \ldots, w_{k}\right\}$ as the eigen $p$-forms of Busemann ball $B((1+\varepsilon) r)$ corresponding to the nonzero eigenvalues

$$
\left\{\lambda_{1}(B((1+\varepsilon) r)), \ldots, \lambda_{k}(B((1+\varepsilon) r))\right\},
$$

we get an orthonormal basis $\left\{v_{1}, \ldots, v_{k}\right\}$ of $\mathscr{V}$ with respect to the inner product $S_{(1+\varepsilon) r}$ satisfying

$$
S_{(1+\varepsilon) r}\left(v_{i}, v_{j}\right)=V^{-1}((1+\varepsilon) r) \int_{B((1+\varepsilon) r)}\left\langle v_{i}, v_{j}\right\rangle .
$$

Hence

$$
\Phi\left(v_{i}, w_{j}\right)=\int_{B((1+\varepsilon) r)}\left\langle v_{i}, w_{j}\right\rangle \phi d v=0
$$

for $1 \leq j<i \leq k$. Thus, for any $1 \leq i \leq k$, let $\left|v_{i}\right|^{2}=\left\langle v_{i}, v_{i}\right\rangle,\left\|v_{i}\right\|^{2}=\left(v_{i}, v_{i}\right)=$ $\int\left\langle v_{i}, v_{i}\right\rangle$ and sgn $=(-1)^{m(p+1)+1}$. We have

$$
\lambda_{i}(B((1+\varepsilon) r)) \int_{B((1+\varepsilon) r)}\left|\phi v_{i}\right|^{2} d v \leq\left\|d\left(\phi v_{i}\right)\right\|^{2}+\left\|\delta\left(\phi v_{i}\right)\right\|^{2} .
$$

The right-hand side of this inequality can be rewritten as

$$
\begin{aligned}
& \left(d\left(\phi v_{i}\right), d\left(\phi v_{i}\right)\right)+\left(\delta\left(\phi v_{i}\right), \delta\left(\phi v_{i}\right)\right) \\
& =\left(d \phi \wedge v_{i}+\phi d v_{i}, d \phi \wedge v_{i}+\phi d v_{i}\right) \\
& +\left(\phi \delta v_{i}+\operatorname{sgn}\left(d \phi \wedge * v_{i}\right), \phi \delta v_{i}+\operatorname{sgn} *\left(d \phi \wedge * v_{i}\right)\right) \\
& =\left\|d \phi \wedge v_{i}\right\|^{2}+2\left(\phi d v_{i}, d \phi \wedge v_{i}\right)+\left\|\phi d v_{i}\right\|^{2}+\left\|\phi \delta v_{i}\right\|^{2} \\
& +2\left(\phi \delta v_{i}, \operatorname{sgn} *\left(d \phi \wedge * v_{i}\right)\right)+\left\|d \phi \wedge * v_{i}\right\|^{2}
\end{aligned}
$$

On the other hand,

$$
\begin{aligned}
0 & =\int_{B((1+\varepsilon) r)} \phi^{2}\left\langle v_{i}, \Delta v_{i}\right\rangle d v=\left(\phi^{2} v_{i}, \Delta v_{i}\right)=\left(\delta\left(\phi^{2} v_{i}\right), \delta v_{i}\right)+\left(d\left(\phi^{2} v_{i}\right), d v_{i}\right) \\
& =\left(\phi \delta v_{i}, \phi \delta v_{i}\right)+2\left(\phi \delta v_{i}, \operatorname{sgn} *\left(d \phi \wedge * v_{i}\right)\right)+\left(\phi d v_{i}, \phi d v_{i}\right)+2\left(\phi d v_{i}, d \phi \wedge v_{i}\right) .
\end{aligned}
$$

Then (22) gives

$$
\begin{aligned}
\left(d\left(\phi v_{i}\right), d\left(\phi v_{i}\right)\right)+\left(\delta\left(\phi v_{i}\right), \delta\left(\phi v_{i}\right)\right) & =\left\|d \phi \wedge v_{i}\right\|^{2}+\left\|d \phi \wedge * v_{i}\right\|^{2} \\
& \leq 2 \sup _{B((1+\varepsilon) r)}|\nabla \phi|^{2} \cdot\left\|v_{i}\right\|^{2} \\
& \leq \frac{8}{\varepsilon^{2} r^{2}} V((1+\varepsilon) r)
\end{aligned}
$$

since $v_{i}$ is orthonormal on $B((1+\varepsilon) r)$. Therefore, (21) and (23) imply 


$$
\begin{aligned}
\int_{B(r)}\left|v_{i}\right|^{2} d v & \leq \int_{B((1+\varepsilon) r)}\left|\phi v_{i}\right|^{2} d v \\
& \leq \lambda_{i}^{-1}(B((1+\varepsilon) r))\left\{\left\|d\left(\phi v_{i}\right)\right\|^{2}+\left\|\delta\left(\phi v_{i}\right)\right\|^{2}\right\} \\
& \leq \frac{8}{\varepsilon^{2} r^{2} \lambda_{i}(B((1+\varepsilon) r))} V((1+\varepsilon) r) .
\end{aligned}
$$

Hence, if we let $Y$ represent the space spanned by $\left\{v_{s+1}, \ldots, v_{k}\right\}$, we get

$$
\operatorname{dim} Y=k-s
$$

and

$$
\sum_{i=s+1}^{k} \int_{B(r)}\left|v_{i}\right|^{2} d v \leq \sum_{i=s+1}^{k} \frac{8}{\varepsilon^{2} r^{2} \lambda_{i}(B((1+\varepsilon) r))} V((1+\varepsilon) r) .
$$

Therefore,

$$
\sum_{i=s+1}^{k} V^{-1}(r) \int_{B(r)}\left|v_{i}\right|^{2} d v \leq \sum_{i=s+1}^{k} \frac{8}{\varepsilon^{2} r^{2} \lambda_{i}(B((1+\varepsilon) r))} \frac{V((1+\varepsilon) r)}{V(r)} .
$$

Moreover, volume doubling property holds on each end of $M$ which implies

$$
V((1+\varepsilon) r) \leq(1+\varepsilon)^{\mu} V(r)
$$

for $r>2 r_{0}$, where $\mu>0$ is a constant depending only on $m$. We conclude

$$
\sum_{i=s+1}^{k} S_{r}\left(v_{i}, v_{i}\right) \leq \frac{8(1+\varepsilon)^{\mu}}{\varepsilon^{2} r^{2}} \sum_{i=s+1}^{k} \lambda_{i}^{-1}(B((1+\varepsilon) r)) .
$$

Lemma 3.3. Let $E_{1}, \ldots, E_{L}$ be the ends of $M$ with respect to $B\left(r_{0}\right), r_{0}>0$, and let $\mathscr{V}$ be a $k$-dimensional vector space with polynomial growth of degree at most $d$. Then for all $0<\varepsilon<\frac{1}{4}$ and $r_{1}>4 r_{0}$, there is $r>r_{1}$ such that if $\left\{u_{1}, \ldots, u_{k}\right\}$ is an orthonormal basis for $\mathscr{V}$ with respect to $S_{(1+\varepsilon) r}$, then

$$
\sum_{i=1}^{k} S_{r}\left(u_{i}, u_{i}\right) \geq k(1+\varepsilon)^{-(2 d+1)} .
$$

Proof. Denote the trace of $S_{r}$ with respect to $S_{(1+\varepsilon) r}$ by $\operatorname{tr}_{(1+\varepsilon) r} S_{r}$. and the determinant of $S_{r}$ with respect to $S_{(1+\varepsilon) r}$ by $\operatorname{det}_{(1+\varepsilon) r} S_{r}$. Suppose the lemma is false. Then, for some $0<\varepsilon<\frac{1}{4}$ and $r_{1}>4 r_{0}$ such that for all $r>r_{1}$,

$$
\operatorname{tr}_{(1+\varepsilon) r} S_{r}=\operatorname{tr}_{(1+\varepsilon) r} S_{r}<k(1+\varepsilon)^{-(2 d+1)} .
$$

On the other hand, the arithmetic geometric mean asserts that

$$
\left(\operatorname{det}_{(1+\varepsilon) r} S_{r}\right)^{1 / k} \leq k^{-1} \operatorname{tr}_{(1+\varepsilon) r} S_{r} .
$$


This implies that

$$
\operatorname{det}_{(1+\varepsilon) r} S_{r} \leq(1+\varepsilon)^{-k(2 d+1)} .
$$

Setting $r=r_{1}+1$ and iterating this inequality $j$ time, we obtain

$$
\operatorname{det}_{(1+\varepsilon)^{j} r} S_{r} \leq(1+\varepsilon)^{-j k(2 d+1)} .
$$

However, for a fixed $S_{r}$ orthonormal basis $\left\{u_{i}\right\}_{i=1}^{k}$ of $\mathscr{V}$, and the polynomial growth assumption imply that there exists a constant $C>0$, depending on $\mathscr{V}$, such that

$$
S_{(1+\varepsilon)^{j} r}\left(u_{i}, u_{i}\right)=V^{-1}\left((1+\varepsilon)^{j} r\right) \int_{B\left((1+\varepsilon)^{j} r\right)}\left\langle u_{i}, u_{i}\right\rangle \leq C\left((1+\varepsilon)^{j} r\right)^{2 d}
$$

for all $1 \leq i \leq k$. Hence

$$
\operatorname{det}_{r} S_{(1+\varepsilon)^{j} r} \leq k ! C^{k}\left((1+\varepsilon)^{j} r\right)^{2 d k} .
$$

This contradicts (25) since $j \rightarrow \infty$.

We are now ready to prove the Main Theorem, which we restate here.

Main Theorem. Let $\left(M^{m}, g\right), m \geq 3$, be a complete noncompact manifold with metric $g$, and $q \in M$ be a fixed point. Suppose curvature operator $K_{p}$ is asymptotically nonnegative. Let $E_{1}, E_{2}, \ldots, E_{L}$ be the ends of $M$ with respect to $B_{q}\left(r_{0}\right)$, $r_{0}>0$. Then for any uniformly equivalent metric $g^{\prime}$ on $M$ and for all $d \geq 1$, the space $H_{d}^{p}\left(M, g^{\prime}\right)$, is finite-dimensional and its dimension satisfies the inequality

$$
\operatorname{dim} H_{d}^{p}\left(M, g^{\prime}\right) \leq C d^{v}
$$

for some constants $v>2, \mu>0, \eta>0$ and $C=C(m, p, v, \mu, \eta)>0$.

Proof. For any $k$-dimensional subspace $\mathscr{V}$ of $H_{d}^{p}(M)$. By Lemma 3.3, if we set $\varepsilon=1 / 5 d$, there exists $r>4 r_{0}$ such that

$$
\operatorname{tr}_{(1+\varepsilon) r} S_{r} \geq k(1+\varepsilon)^{-(2 d+1)} .
$$

Let $\lambda_{k}$ be the $k$-th eigenvalue of the Hodge Laplacian acting on $p$-forms on Busemann ball $B((1+\varepsilon) r) \supset B\left(r_{0}\right)$ satisfying the absolute boundary condition on $\partial B((1+\varepsilon) r)$ under the metric $g^{\prime}$. Then by Lemma 2.1, Lemma 2.2 and the assumption of $K_{p}$, we have

$$
\lambda_{k} \geq C k^{2 / v}(1+\varepsilon)^{-2} r^{-2},
$$

for $k>s=\sum_{l=1}^{L} \operatorname{dim} H_{p}(B((1+\varepsilon) r))$, where $C$ is a positive constant depending only on $m, p, v$ and $\eta$. Combining with Lemma 3.2, we find there exists a subspace $Y$ in $\mathscr{V}$ with

$$
\operatorname{dim} Y=\operatorname{dim} \mathscr{V}-\sum_{l=1}^{L} \operatorname{dim} H_{p}(B((1+\varepsilon) r)),
$$


and a positive constant $\mu$ such that

$$
\sum_{i=s+k_{0}+1}^{k} S_{r}\left(v_{i}, v_{i}\right) \leq \frac{8(1+\varepsilon)^{\mu}}{\varepsilon^{2} r^{2}} \sum_{i=s+1}^{k} \lambda_{i}^{-1}(B((1+\varepsilon) r)) \leq \frac{8(1+\varepsilon)^{\mu+2}}{\varepsilon^{2}} k^{1-2 / \nu},
$$

where $\varepsilon=1 / 5 d$. Hence

$$
\begin{aligned}
k(1+\varepsilon)^{-(2 d+1)} & \leq \operatorname{tr}_{(1+\varepsilon) r} S_{r}(V) \leq \operatorname{tr}_{(1+\varepsilon) r} S_{r}(Y)+\operatorname{dim} H_{p}(B((1+\varepsilon) r)) \\
& \leq C d^{2} k^{1-2 / \nu} .
\end{aligned}
$$

where we have used Lemmas 2.1 and 2.2. Therefore $k \leq C d^{v}$. Since $\mathscr{V}$ is arbitrary, we conclude that

$$
\operatorname{dim} H_{d}^{p}\left(M, g^{\prime}\right) \leq C d^{v}
$$

for all $d \geq 1$.

Suppose $M$ has nonnegative curvature operator outside a compact set, with finite first Betti number, In this case, even thought it is not exactly true that each end of $M$ satisfies volume comparison property for $p=1$, however, it is almost true so that by modifying some arguments of main theorem still holds for such a manifold; see [Li and Tam 1995, Corollary 6.2] in particular. Hence we have Corollary 1.3.

\section{Acknowledgement}

We thank Professor Jiaping Wang for his interest in this work.

\section{References}

[Chen and Sung 2006] J.-T. R. Chen and C.-J. A. Sung, "Dimension estimate of polynomial growth harmonic forms", J. Differential Geom. 73:1 (2006), 167-183. MR 2007b:53158 Zbl 05042956

[Dodziuk 1982] J. Dodziuk, "Eigenvalues of the Laplacian on forms", Proc. Amer. Math. Soc. 85:3 (1982), 437-443. MR 84k:58223 Zbl 0502.58038

[Donnelly and Li 1982] H. Donnelly and P. Li, "Lower bounds for the eigenvalues of Riemannian manifolds", Michigan Math. J. 29:2 (1982), 149-161. MR 83g:58069 Zbl 0488.58022

[Hajłasz and Koskela 1995] P. Hajłasz and P. Koskela, "Sobolev meets Poincaré", C. R. Acad. Sci. Paris Sér. I Math. 320:10 (1995), 1211-1215. MR 96f:46062 Zbl 0837.46024

[Li 1980] P. Li, "On the Sobolev constant and the p-spectrum of a compact Riemannian manifold", Ann. Sci. École Norm. Sup. (4) 13:4 (1980), 451-468. MR 82h:58054 Zbl 0466.53023

[Li 1997] P. Li, "Harmonic sections of polynomial growth", Math. Res. Lett. 4:1 (1997), 35-44. MR 98i:53054 Zbl 0880.53039

[Li and Tam 1992] P. Li and L.-F. Tam, "Harmonic functions and the structure of complete manifolds”, J. Differential Geom. 35:2 (1992), 359-383. MR 93b:53033 Zbl 0768.53018

[Li and Tam 1995] P. Li and L.-F. Tam, "Green's functions, harmonic functions, and volume comparison”, J. Differential Geom. 41:2 (1995), 277-318. MR 96f:53054 Zbl 0827.53033

[Li and Wang 1999] P. Li and J. Wang, "Counting massive sets and dimensions of harmonic functions”, J. Differential Geom. 53:2 (1999), 237-278. MR 2001k:53063 Zbl 1039.53036 
[Saloff-Coste 1992] L. Saloff-Coste, "Uniformly elliptic operators on Riemannian manifolds", $J$. Differential Geom. 36:2 (1992), 417-450. MR 93m:58122 Zbl 0735.58032

[Tam 1998] L.-F. Tam, "A note on harmonic forms on complete manifolds", Proc. Amer. Math. Soc. 126:10 (1998), 3097-3108. MR 99a:53047 Zbl 0922.58017

[Yau 1975] S. T. Yau, "Harmonic functions on complete Riemannian manifolds", Comm. Pure Appl. Math. 28 (1975), 201-228. MR 55 \#4042 Zbl 0291.31002

Received April 17, 2006.

JUI-TANG RAY CHEN

DEPARTMENT OF MATHEMATICS

NATIONAL TSING HUA UNIVERSITY

HSINCHU

TAIWAN 30013

jtchen@math.nthu.edu.tw

ChiUng-Jue AnNa Sung

DEPARTMENT OF MATHEMATICS

National TSING Hua University

HSINCHU

TAIWAN 30013

cjsung@math.nthu.edu.tw www.math.nthu.edu.tw/ cjsung 